\title{
Image Tampering Detection Based on Combined Feature Extraction Methods
}

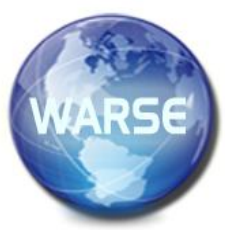

\begin{abstract}
Image manipulated identification is varied from longstanding old semantic location since it demonstrates more arrangement to intruding relics than to picture material, which recommend that favored highlights should be illuminated. We propose a cross breed highlight model like convolutional neural Network System (CNN), histogram of situated slope (HOG), scale-invariant component change (sift) highlights to identify the altered areas given a controlled picture. Initially, we propose the SIFT based alter area location. At that point the framework contains the information picture is portioned into covering squares, whereupon a HOG-CNN highlight is applied to each square. HOG - CNN highlights are removed and decreased to encourage the estimation of closeness. At long last, highlight vectors are lexicographically arranged, and altered picture alter district squares are distinguished by K-Nearest Neighbor. Finally, joining of SIFT based alter area location result and HOG-CNN based alter locale recognition result. Investigation yields show that the proposed way can distinguish different instances of numerous kinds of duplicate and move , grafting imitation, expulsion fraud and precisely find the twinned replicated areas, in any event, when taking care with pictures distorted by interpretation including any little changes like revolutions, obscuring, modifying the brilliance, and shading changes.
\end{abstract}

Key words: Histogram of oriented Gradients, Convolutional Neural Networks, Scale Invariant Feature Transform, Computer Vision.

\section{INTRODUCTION}

Image processing is a system to change over an image into computerized frame and play out certain activities subsequently, in order to ask an improved picture or to separate some helpful data from it. A subsection of sign allotment, image processing allotment ensures that information is picture. Additionally, similar to video edge or photo and yield could likewise be picture or attributes identified with that picture. Generally, Image Processing structure will in general view pictures as bi-dimensional signs while applying starting at now arranged sign taking care of frameworks to them.

It is one of the fast-changing progressions nowadays, with its uses in various areas of business. Picture Processing structures center research region inside building and figuring disciplines as well. In this manner, picture handling essentially incorporates the accompanying three stages:

1. Using computerized photography such as optical scanner for the purpose of importing images.

2. Investigating and controlling the image which "consolidates data weight and picture and spotting structures" those are not to regular eyes like satellite photographs.

3. The respected result can be altered and manipulated in the last stage that comprises of the output. This stage depends on picture investigation.

\section{RELATED WORK}

In order to exploit spatial structure for localizing manipulated image regions. The appearance of innovative journaling apparatuses encourages an image to be controlled during a way which will handily dodge best in class picture altering discovery draws near. The ongoing achievement of the profound learning approaches in a few acknowledgment assignments rouses us to build up a high certainty recognition system which may confine controlled areas in an image. Unique in relation to semantic item division where each significant district is sectioned, the limitation of picture control concentrates just the conceivable altered area which makes the issue considerably harder. In this way, to plan the system, we apply a half and half CNN-LSTM model to get significant highlights among controlled and noncontrolled areas. One of the fundamental highlights of controlled locales is that they show discriminate highlights in limits imparted to 
neighbouring non-controlled pixels. Our inspiration is to "discover the limit disparity, i.e., the spatial structure, among controlled and noncontrolled territories with the mix of LSTM" and convolution layers. We perform start to finish preparing of the system to get familiar with the parameters through back propagation given ground-truth cover data. The general structure is fit for distinguishing varying kinds of picture controls, including duplicate move, expulsion and joining.

Our model demonstrates promising prompts restricting controlled locales, which is exhibited through thorough experimentation on three differentdata sets [1]. A deep learning approach to universal image manipulation detection using a new convolutional layer has been used in this work [2]. While making a fraud, a controller can adjust an image utilizing numerous different picture-altering activities. Since a scientific inspector must test for everything about, noteworthy intrigue has emerged inside the improvement of all-inclusive measurable calculations fit for recognizing numerous different picture- altering activities and controls. In this paper, they propose a comprehensive quantifiable approach to manage performing control acknowledgment using significant learning.

In particular, we propose new convolutional determination prepared to do naturally gaining control discovery includes legitimately from preparing information. In their present structure, convolutional neural systems will learn highlights that catch a picture's substance as against control identification highlights. To dispose this issue, we made a substitution kind of convolutional layer that is explicitly organized to stifle a picture's substance and adapt learn phony discovery highlights. Subsequent to taking out numerous tests, it portrayed that the proposed method can consequently discover how to identify various picture controls without depending on already chosen highlights or any pre-processing. The yield of those investigations express that our proposed way can naturally distinguish a few distinct controls with a mean precision of 99.10\%.Recasting residual-based local descriptors as convolutional neural networks: an application to image forgerydetection[3].Local descriptors upheld the picture clamour leftover have demonstrated incredibly compelling for assortment of scientific applications, similar to fabrication discovery and limitation.

In any case, inspired by promising prompts PC vision, the primary objective of the exploration network is presently moving on profound learning. In this paper, they show that a class of waiting based descriptors can be truly seen as an essential constrained" convolutional neural network. At that point, by loosening up the imperatives, and tweaking web on a nearly little preparing set, we get a major presentation improvement regarding the conventional indicator. Single-image splicing localization through auto encoder-based anomaly detection [4]. Here, they propose a swap technique for single picture joining restriction. Without any side data, such as preparing information or earlier information on thesource camera and in this manner picture history, we give the issue a role as an inconsistency identification task. Expressive neighbourhood highlights, separated from the clamour leftover of the picture, which produces a verifiable model of the information.

By rehashing, discriminant trademark marking and auto encoding, the certain model fits in the long run the flawless information, while the joined area is perceived as irregular. Experiments on a fitting test set of joined images show that the proposed methodology beats the past top tier. Likewise, it displays a decent power against commonplace social net post-preparing, demonstrating guarantees for genuine applications.

Image splicing localization using a multitask fully connected convolutional network.In this work, they propose a way that uses an absolutely convolutional arrange (FCN) to confine picture joining assaults [5]. As a matter of first importance, single-task FCN (SFCN) prepared uniquely on a superficial level mark is assessed. Disregarding the way that the SFCN is seemed to supply better execution over existing procedures, it regardless of everything gives a cruel limitation yield in explicit cases. Thus, we propose the "utilization of a play out various errands FCN (MFCN) that utilizes two yield branches for play out" different assignments learning. One branch is utilized to discover the surface mark, while the contrary branch is utilized to discover the sting or limit of the grafted locale. They prepared it so that using various data sets. Trials that occurred says that the SFCN and MFCN outflank previously existing grafting restriction calculations, which the MFCN can do better confinement than theSFCN [6].

In Existing works, the computational speed is low. The rate of tamper region detection accuracy is less. Some existing models are there which doesn't detect the three-image manipulation. It detects only one image manipulation $[7,8]$.

Despite this classification, the detection is not fullproof. This method is independent of any unique criterion. These overlapping in its result and the lack of a unique algorithm for classification are the main drawback of the existing model [9].

\section{PROPOSED WORK}

In order to overcome the challenges listed in the survey. The following steps are carried out in this proposed system

Step 1: To get the original image and tamper image from the public database. 
Step 2: To find the tamper region using SIFT method.

Step 3: By applying some algorithms for tamper region detection to enhance the accuracy of image manipulation detection,

Step 4: We propose the block separation process to both images. Next, extracting the rich image features for each block of both images based on histogram of oriented gradients convolutional neural network.

Step 5: Machine learning model is proposed to each block of authentic and tamper image features for detect the tamper region in tampered image. Here $\mathrm{KNN}$ is applied for similarity measurement.

Step 6: Final tamper region detection done by hybrid feature model i.e. combining of SIFT-HOGCNN. This makes the model more effective to do copy-move, splicing and removal regiondetection [10].

\section{IMPLEMENTATION}

Convolutional neural networks used for rich highlights extraction. SIFT likewise used to identify the altered picture locale. Hog feature which is similarly utilized with CNN which is an element descriptor for image preparing with the last goal of its position [11-13].K-Nearest Neighbor utilized for square coordinating dependent on square CNN highlights.

\section{A. Image Procurement}

Image procurement while doing picture handling can be characterized as the "demonstration of getting a picture from any source, generally an equipment-based origin, so it very well may be gone through" whatever procedures need to happens short time later. Doing picture procurement while picture preparing is consistently the initial step of the work process succession on the grounds that, without a picture, no handling is conceivable. The picture that we get isn't handled and is the yield of "whatever equipment was utilized to get it, which can be significant" in certain fields to have a steady benchmark from which to work. Authentic and altered pictures are gained from exhibition [14, $15]$.

\section{B. Tamper Region Detection by SIFT}

In this module, we used SIFT features for tamper region detection. We first distinguish key focuses on the picture dependent on the DOG indicator. A short time later, the SIFT descriptor is picked for key point portrayal. The mix of DOG locator and SIFT descriptor has been appeared to beat other indicator and descriptor blends in numerous applications. SIFT feature extraction is applied to both images i.e. authentic image and tampered image. Finally, based on the matched key points tamper regions are identified [16, 17].

\section{Tamper Region Detection by HOG-CNN}

BLOCK SEPARATION: After image acquisition, we implement the block separation module to authentic and tamper images. In both images, block separation is implemented. Totally we get 16 blocks from whole image.

FEATURE EXTRACTION: In highlight extraction, we separate took in rich picture highlights from a pre-trained convolutional neural system and histogram of situated inclinations and utilize those highlights to alter area recognition. Feature extraction is the least demanding and quickest approach to utilize the authentic intensity of pre-trained profound systems and hoard.

\section{CNN Based Feature Extraction}

For properties extraction, we propose the ResNet50 CNN model [18]. In this system makes a various leveled portrayal of info pictures. Profound layers incorporate more elevated level highlights, made utilizing the lower-level highlights of before layers. To get the element portrayals of each square of the two pictures, use enactments on the worldwide pooling layer, 'pool5', toward the finish of the system. The worldwide pooling layer pools the highlights over every single spatial area, giving the effective highlights. HOG descriptor is for the most part appropriate for alter locale identification in pictures because of some key points of interest contrasted with different descriptors.[19]Firstly, it takes a shot at neighborhood cells, so it is invariant to geometric and photo metric changes. Also, at that point the coarse (spatial) testing, fine direction examining, and solid neighborhood photometric standardization permit diverse body development of pictures to be disregarded in case they keep up a by and large upstanding position[20,21].

\section{E. HOG Based Extraction}

First, the picture is partitioned into little locales which are associated together which are called cells and these phones register a histogram of edge directions or histogram of inclination headings for every pixel which is available inside the phone Then as indicated by the angle direction, every cell is discretized into precise canisters[22,23].

- Angular receptacle weighted angle is contributed by each relating cell's pixel.

- It builds square which is a gathering of contiguous cells.

- The premise of standardization of histograms is the above gathering of cells in squares.

- This structure a square histogram which is a standardized gathering of histograms which speaks to a descriptor that is a lot of these square histograms [24]. 


\section{F. TAMPER REGION DETECTION}

In this module, block matching is performed to detect the tamper region in tampered image. For block matching, machine learning model is KNN used. Block matching done using $\mathrm{KNN}$ based on block HOG-CNN features. Finally, based on the matching result tamper regions are identified [25].

\section{RESULTS AND DISCUSSION}

The system takes the original image as input and outputs the exact area where the image tampered. Also it gives us more accurate results because it using HOG followed by SIFT and then KNN classification.The Figure.1 shows the features extracted using CNN and Hog individually.

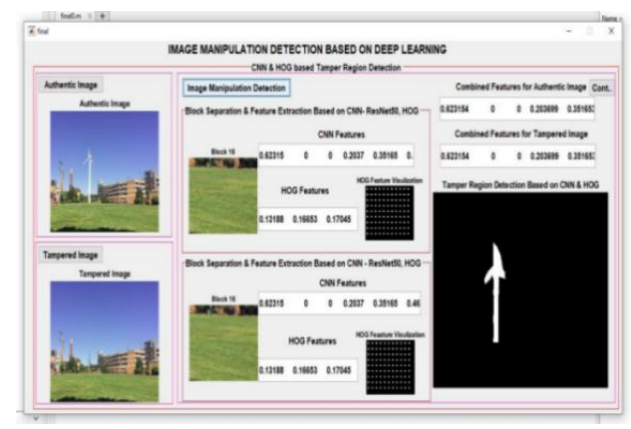

Figure1: Hog and CNN Based Detection

In Figure 2 the fusion results of SIFT based Tamper Region, CNN-Hog based Tamper Region and proposed Tamper Region based on SIFTCNN-HOG.

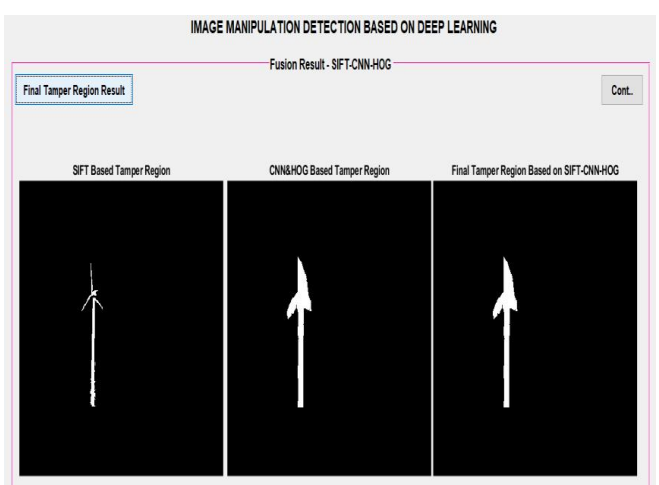

Figure 2: Fusion Result of SIFT-CNN-HOG

In the Figure 3 and Figure 4 shows the original image and the tampered region. It shows better results compared to other existing works.
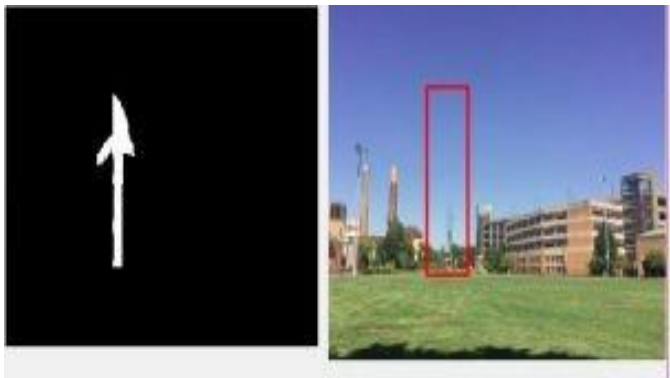

Figure 3: Original Image

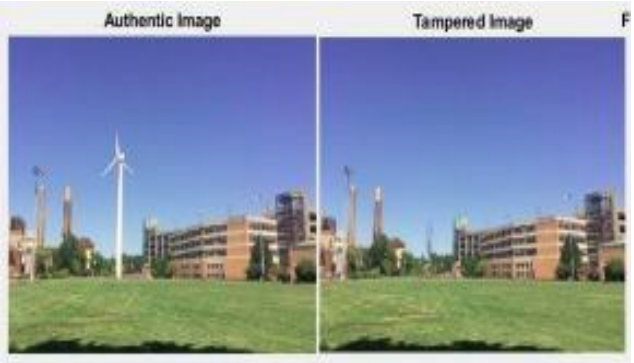

Figure 4: Result Exhibiting tampered Region

\section{CONCLUSION}

The paper gives an exclusive way of Here, we present a method using an RGB stream" to learn rich features for image manipulation detection. Image manipulation detection followed as the first input image is segmented into overlapping blocks. Second, CNN features are applied to each block with that HOG is used and SIFT is also considered to get the tamper region; finally, machine learning model like $\mathrm{KNN}$ is proposed to detect the tamper region based on the fusion result of the SIFT-CNN-HOG. Our proposed model is applicable to three applications like copy move forgery detection, splicing detection and removal detection with great accuracy. Thus, our proposed model provided better performance than the existingmodels.

\section{REFERENCES}

[1] J. H. Bappy, A. K. Roy-Chowdhury, J. Bunk, L. Nataraj, and B. Manjunath. "Exploiting spatial structure for localizing manipulated image regions". In ICCV, 2017.

[2] B. Bayar and M. C. Stamm. "A deep learning approach to universal image manipulation detection using a new convolutional layer". In IH \& MMSec,2016.

[3] T. Bianchi, A. De Rosa, and A. Piva. "Improved det coefficient analysis for forgery localization in jpeg images". In ICASSP,2011. 
[4] J. Chen, X. Kang, Y. Liu, and Z. J. "Wang. Median filtering forensics based on convolutional neural networks". Signal Processing Letters, 2015.

[5] D. Cozzolino, D. Gragnaniello, and L. Verdoliva. "Image forgery localization through the fusion of camera-based, feature-based and pixel-based techniques".InICIP,2014.

[6] X. Pan, X. Zhang, and S. Lyu. "Exposing image splicing with inconsistent local noise variances". In ICCP, 2012 .

[7] D. Cozzolino, G. Poggi, and L. Verdoliva. Splicebuster: "A new blind image splicing detector". In WIFS, 2015.

[8] D. Cozzolino, G. Poggi, and L. Verdoliva. "Recasting residual-based local descriptors as convolutional neural networks: an application to image forgery detection". In IH\&MMSec, 2017.

[9] D. Cozzolino and L. Verdoliva.'Single-image splicing localization through autoencoderbased anomaly detection". In WIFS, 2016.

[10] J. Dai, Y. Li, K. He, and J. Sun. R-fen: "Object detection via region-based fully convolutional networks". In NIPS,2016.

[11] T.J. DeCarvalho, C.Riess, E.Angelopoulou, H.Pedrini, and A. de Rezende Rocha. "Exposing digital image forgeries by illumination color classification". TIFS, 2013.

[12] J. Dong,W.Wang, and T. Tan. Casia image tampering detection evaluation database 2010. http://forensics.idealtest.org.55

[13] J. Dong, W. Wang, and T. Tan. "Casia image tampering detection evaluation database". In ChinaSIP,2013.

[14] P. Ferrara, T. Bianchi, A. De Rosa, and A. Piva. "Image forgery localization via finegrained analysis of artifacts". TIFS,2012.

[15] J. Fridrich and J. Kodovsky.'Rich models for steganalysis of digital images". TIFS, 2012.

[16] M. Gao, R. Yu, A. Li, V. I. Morariu, and L. S. Davis.'Dynamic zoom-in network for fast object detection in large images". In CVPR,2018.

[17] Y. Gao, O. Beijbom, N. Zhang, and T. Darrell. “Compact bilinear pooling”. In CVPR, 2016.

[18] R. Girshick. Fast r-cnn. In ICCV,2015.

[19] M. Goljan and J. Fridrich. "Cfa-aware features for steganalysis of color images". In SPIE/IS\&T Electronic Imaging, 2015.

[20] K. He, X. Zhang, S. Ren, and J. Sun. "Deep residual learning for image recognition”. In CVPR, 2016

[21] N. Krawetz."A picture's worth", Hacker Factor Solutions, 2007.

[22] T.-Y. Lin, M. Maire, S. Belongie, J. Hays, P.
Perona, D. Ramanan, P. Doll'ar, and C. L. Zitnick. Microsoft coco: "Common objects in context”. In ECCV,2014.

[23] T.-Y. Lin, A. RoyChowdhury, and S. Maji. "Bilinear cnn modelsforfinegrainedvisualrecognition". InICCV,2015.

[24] B. Mahdian and S. Saic. "Using noise inconsistencies for blindimageforensics".ImageandVisionComp uting,2009.

[25] T.-T. Ng, J. Hsu, and S.-F. Chang. Columbia image splicing detection evaluation dataset : http://www.ee.columbia.edu/ln/dvmm/downl oads/AuthSplicedDataSet/AuthSplicedDataS et.htm 